\title{
Oral use of Streptococcus salivarius KI 2 in children with secretory otitis media: preliminary results of a pilot, uncontrolled study
}

This article was published in the following Dove Press journal:

International Journal of General Medicine

15 September 2015

Number of times this article has been viewed

\author{
Francesco Di Pierro' \\ Daniele Di Pasquale ${ }^{2}$ \\ Maurizio Di Cicco² \\ 'Velleja Research, Milan, Italy; ${ }^{2} \mathrm{ORL}$ \\ Department, Ospedale Maggiore \\ Policlinico Ca' Grande IRCCS, \\ Milan, Italy
}

\begin{abstract}
Secretory otitis media (SOM) remains a common disease among children. Although its cause is not yet perfectly established, the pathology, often a sequel of acute otitis media $(\mathrm{AOM})$, is mainly characterized by persistent fluid in the middle ear cavity. Twenty-two children with a diagnosis of SOM were treated daily for 90 days with an oral formulation containing the oral probiotic Streptococcus salivarius K12 (Bactoblis ${ }^{\circledR}$ ). After treatment, the children were evaluated for AOM episodes and subjected to tone audiometry, tympanometry, endonasal endoscopy, otoscopy, and tonsillar examination. Subject compliance and probiotic tolerability and side effects have also been evaluated. Our results indicate a good safety profile, a substantial reduction of AOM episodes, and a positive outcome from the treatment for all of the clinical outcomes tested. We conclude that strain $\mathrm{K} 12$ may have a role in reducing the occurrence and/or severity of SOM in children. From our perspective, this study constitutes a starting point toward the organization of a more extensive placebo-controlled study aimed at critically appraising our preliminary observations.
\end{abstract}

Keywords: BLIS K12, Bactoblis ${ }^{\circledR}$, acute otitis media, exudative otitis media

\section{Introduction}

Otitis media is one of the most frequent problems that must be addressed by physicians dealing with pediatric patients. Approximately $80 \%$ of children have at least one episode of acute otitis media (AOM), and between $80 \%$ and $90 \%$ of preschool children have at least one episode of secretory otitis media (SOM). ${ }^{1,2}$ In AOM, bacterial pathogens such as Streptococcus pneumoniae, Haemophilus influenzae, Moraxella catarrhalis, and Streptococcus pyogenes ascend through the eustachian tube from the nasopharynx to the middle ear, causing an inflammatory response. ${ }^{3}$ SOM, asymptomatic persistence of effusion in the middle ear cavity, is a possible sequel of AOM. SOM often resolves spontaneously but in some cases, especially when bilateral exudate persists for more than 3 months, the insertion of a tympanostomy tube may be required for drainage to avoid hearing difficulties and/or chronic anatomical damage of the tympanic membrane. ${ }^{4}$ Antibiotics, decongestants, and corticosteroids fail to eliminate middle ear exudation and therefore are not recommended. ${ }^{5,6}$ It has been observed that otitisprone children carry more bacterial pathogens in their nasopharyngeal microbiota and fewer potentially-interfering microorganisms such as alpha-hemolytic streptococci, nonhemolytic streptococci, Prevotella and Peptostreptococcus species. ${ }^{7,8}$ Some strains of alpha-hemolytic streptococci depress the growth of pathogenic bacteria in vitro ${ }^{9}$ and nasal spraying with alpha-hemolytic streptococci in otitis-prone children reduces the risk of recurrence of AOM and the development of SOM. ${ }^{10}$ Also, spray treatment with
Correspondence: Francesco Di Pierro Velleja Research, Viale Lunigiana 23, 20125 Milan, Italy

Tel +393495527663

Email f.dipierro@vellejaresearch.com 
alpha-hemolytic streptococci in children with established SOM can diminish the amount of fluid in the middle ear, thus reducing hearing problems and rendering surgery unnecessary in many cases. ${ }^{11}$ Many oral commensal streptococci have been recently investigated for their ability to interfere with the growth of pathogens inhabiting the oral cavity and/ or nasopharynx. ${ }^{12}$ Of these, the oral probiotic Streptococcus salivarius $\mathrm{K} 12$ has been most thoroughly studied. Originally isolated from the throat of a New Zealand child, strain K12 produces two distinct megaplasmid-encoded lantibiotics, named salivaricin A2 and salivaricin B, that inhibit the growth of $S$. pyogenes, $S$. pneumoniae, and $M$. catarrhalis, all of which are involved in the pathogenesis of AOM and bacterial pharyngotonsillitis in children and adults. ${ }^{13-17}$ Strain K12 typically colonizes the oral cavity of more than $30 \%$ of children after 3 days of administration, with clear colonization of even the nasopharynx and adenoids detected for up to 32 days after the last administration. ${ }^{18,19}$ The K12 strain is also endowed with an excellent antibiotic-sensitivity profile and high safety-assurance characteristics, as demonstrated in tests in laboratory animals and humans. ${ }^{20,21}$ Recent clinical trials ${ }^{22-24}$ conducted both in adults and children demonstrated that treatment with the strain $\mathrm{K} 12$ reduces recurrences of bacterial pharyngotonsillitis and AOM. The present preliminary and uncontrolled study, performed in children diagnosed with recurrent $\mathrm{AOM}$ and also affected by asymptomatic SOM, was therefore designed first to evaluate the safety and tolerability profile of strain K12 when administered in children with clear presence of a middle ear exudate, second to establish the possible protective effect in terms of reduction of AOM recurrences, and third to follow the progression of SOM by using tone audiometry, tympanometry, endonasal endoscopy, otoscopy, and tonsillar examination.

\section{Materials and methods}

\section{Subjects}

Twenty-two children (3-9 years old) having a recent history of recurrent AOM and with unilateral or bilateral fluid in the middle ear for at least 2 months were included in this preliminary, uncontrolled study after informed consent was obtained from their parents. Exclusion criteria were severe underlying disease, immune deficiency, heart disease or congenital heart defects, sore mucosae, antibiotic use within the last month, and upper respiratory tract infection in the 10 days preceding the enrollment.

\section{Study scheme}

This pilot, uncontrolled study was conducted in the field of routine clinical practice in the area of Milan (Italy) between November 2013 and September 2014, in agreement with the criteria set by the Declaration of Helsinki. As the product being tested is a nutraceutical, the approval from the ethical board was not required. The parents of all the participants in the study were informed of the trial methods and signed the consent and privacy policy documents giving the authorization to publish the results. The subjects were followed for 2 months before entering the study. At the enrollment $(T=0)$ and after 90 days, all the subjects underwent to physical examination, pharyngeal buffer (Test Strep-A; Gima, Gessate, Italy), bilateral tone audiometric testing, bilateral tympanometry, bilateral otoscopy, endonasal endoscopy, and tonsillar examination (Figure 1). Every 15 days, for the whole duration of the study, all of the enrolled subjects were in contact with the physician responsible for the study to report their medical condition and specific study parameters such as probiotic tolerability and dosing compliance as well as to enable documentation of the occurrence of any

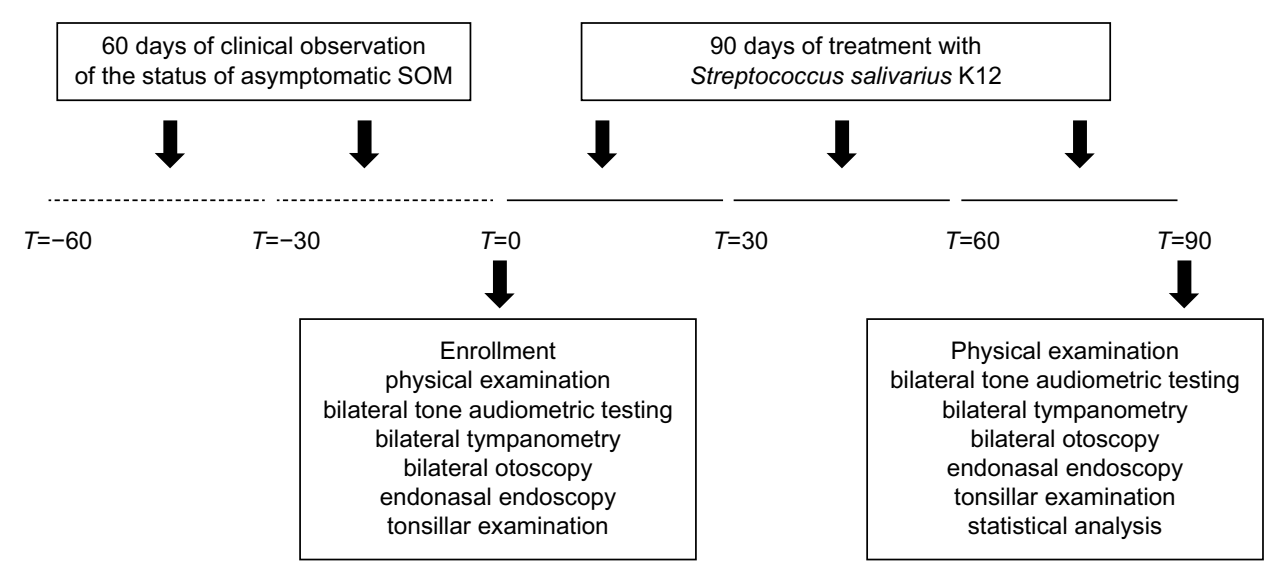

Figure I Scheme of the study.

Abbreviation: SOM, secretory otitis media. 
side effects possibly linked to the treatment. The subjects were also provided with the possibility of daily access to the physician responsible for the study. The treatment was for 3 months from $T=0$ to $T=90$. All 22 enrolled subjects completed the study, and statistical analysis was performed on all subjects.

\section{Tested product}

S. salivarius K12, also known as BLIS K12 (BLIS Technologies, Dunedin, New Zealand), was formulated as slowlydissolving oral tablets by SIIT (Trezzano, Milan, Italy) and notified as nutritional supplement to the Italian Ministry of Health as Bactoblis ${ }^{\circledR}$ by Omeopiacenza (Pontenure, Italy), according to the provisions of law 169 of 2004, on July 5, 2011 (notification number 53435). The preparation of Bactoblis ${ }^{\circledR}$ used in the clinical trial contained no less than 1 billion colony-forming units/tablet of $S$. salivarius K12.

\section{Treatment protocol}

Starting from $T=0$ to $T=90,1$ tablet of Bactoblis ${ }^{\circledR}$ was administered to each subject every night, just before sleep. The tablet was allowed to slowly dissolve in the oral cavity, without biting or swallowing. Saliva production is typically reduced in the evening hours and this improves the effectiveness of oral colonization. Only for the very first treatment, the administration of the tablet was preceded, approximately 30 minutes before, by the use of a chlorhexidine-based $(0.2 \%)$ mouthwash. This procedure improves the efficacy of oral colonization by BLIS K12 by creating bacteria-depleted niches in the oral tissues. In order to evaluate the level of subject adherence to the established protocol, the subjects were asked to return any unused product boxes and tablets. Acceptable adherence was considered to be the administration of not less than $95 \%$ of the allocated tablets.

\section{Study objectives}

The study aims were to evaluate: 1) the safety profile of S. salivarius K12 when administered for 90 days to children with a previous diagnosis of recurrent $\mathrm{AOM}$ and in presence of middle ear exudate for at least 2 months; 2) the capability of $S$. salivarius K12 to protect against AOM recurrence; 3 ) to follow the possible evolution of SOM by using tone audiometry, tympanometry, endonasal endoscopy, otoscopy, and tonsillar examination.

\section{AOM incidence}

The incidence of AOM has been calculated as episodes of AOM per month per child. The value of AOM incidence described at $T=0$ corresponds to the one calculated according to the episodes occurred the previous 12 months. The value of AOM incidence described at $T=90$ corresponds to the one calculated according to the episodes occurred during the 90 days of treatment.

\section{Audiometry}

AM13 FreeQuency (Tecnomed) audiometer, TDH 39 (Telephonics) headphones, and a soundproof booth (Mitaso) were used. Pure tone audiometry (air conduction) was performed at frequencies ranging from 250 to $8,000 \mathrm{~Hz}$ for the hearing threshold test. When necessary the bone route (frequencies of 250 to $4,000 \mathrm{~Hz}$ ) was applied. The technique used was sound-to-silence, and the threshold was considered to be the lowest intensity at which the child responded $100 \%$ of the times to the presence of sound. To classify the degree, we used the mean tonal thresholds per airway at the frequencies of $250-2,000 \mathrm{~Hz}$ and values proposed by Northern and Downs $^{25}$ for children classifying as normal (score $=0$; up to $15 \mathrm{~dB}$ ); mild transmissive hypoacusis ( to $70 \mathrm{~dB}$ ); severe transmissive hypoacusis ( more than $71 \mathrm{~dB}$ ).

\section{Tympanometry}

A Zodiac 901 tympanometer (Madsen Electronics, Taastrup, Denmark) was used to assess the condition of the middle ear. The tympanogram was evaluated according to three types of conditions: type A (normal); type B (presence of middle ear exudate); type $\mathrm{C}$ (tubaric dysfunction).

\section{Otoscopy}

The data obtained from otoscopy were categorized into four grades of classification: normal tympanic membrane ( score $=0)$; matt-like tympanic membrane (score $=1$ ); mattlike and retracted tympanic membrane (score $=2)$; and adherent (glue-ear) tympanic membrane (score $=3$ ).

\section{Endonasal endoscopy}

Endonasal endoscopic examination was done using an Olympus pediatric fiberscope with a $2.2 \mathrm{~mm}$ flexible nasal endoscope. The other equipment used for assessing slow movements were video camera attached to the endoscope, a colored television, and an image recorder. Data have been evaluated as percentage of obstruction.

\section{Tonsillar examination}

Tonsil volume was classified according to validated criteria ${ }^{26}$ as follows: tonsils in the tonsillar fossa barely seen behind the 
anterior pillar (score $=0$ ); tonsils visible behind the anterior pillar (score $=1$ ); hypertrophic tonsils extended three-quarters of the way to middle line ( score $=2$ ); and tonsils completely obstructing the airway, known as kissing tonsils (

\section{Statistical analysis}

The difference between the two groups of clinical variables, pre- and posttreatment with $S$. salivarius K12, was determined using the two-tailed Wilcoxon-Mann-Whitney test. Only for endonasal endoscopy, since it is a ratio variable, we transformed the data with arcsin function before applying the Wilcoxon-Mann-Whitney test. The difference between pre- and posttympanometry data was determined using the Fisher's exact test. Statistical software used was JMP ${ }^{\circledR} 10$ for Mac OS X (SAS Institute, Cary, NC, USA), and the threshold for statistical significance was $95 \%$.

\section{Results}

In our study, 22 children aged between 3 and 9 years were enrolled. At the end of the study, all were considered eligible for statistical analysis, having completed the study according to the protocol. In Table 1, sex, age, weight, height, body mass index, and other clinical characteristics of the enrolled subjects are reported. Among the 22 enrolled subjects, eight reported a history of allergy, 20 had been breastfed for at least 4 months since delivery, and 20 had been vaccinated with 13-valent pneumococcal conjugate vaccine. At enrollment, four of the 22 subjects were swab-test positive but asymptomatic for $S$. pyogenes. The same four subjects were also swab-test positive and asymptomatic at the end of the study, indicating that they were healthy carriers. At the end of the study, no other subjects were swab-test positive for $S$. pyogenes. As shown in Table 2, the oral use of S. salivarius K12 appears to have modified some clinically relevant outcomes. AOM incidence, calculated as the number of episodes per month per child, was reduced by approximately $40 \%$ during the 3 months of treatment as compared with the corresponding incidence calculated according to the number of episodes that occurred in the previous year. Pure tone audiometry improved by more than 50\% (left ear) and $60 \%$ (right ear), and, otoscopy, bilaterally, by approximately
$40 \%$. Eustachian tube obstructions due to adenoid vegetation hypertrophy decreased by approximately $30 \%$. Collectively, the sizes of palatine tonsils were reduced by $40 \%$, and only two cases of kissing tonsils were detected at $T=90$ by comparison to ten cases at $T=0$ (data not shown). As shown in Table 3, tympanometry was substantially improved, particularly concerning the presence of middle ear exudate which occurred at $T=90$ in only two cases, bilaterally. No relevant differences were observed concerning tubaric dysfunction. Finally, the probiotic treatment demonstrated a very good safety profile with no treatment-related side effects occurring and no subject drop out. As shown in Table 4, tolerability was assessed as "good" and "very good" in 20 of the 22 subjects and overlapping results were obtained as regards to compliance.

\section{Discussion}

In a previous study, a nasal spray bacterial treatment was shown to reduce the development of SOM as a sequel to AOM in otitis-prone children. ${ }^{10}$ In a later study, it was shown that a spray treatment with alpha-hemolytic streptococci led to complete or almost complete resorption of middle ear effusion in one-third of treated patients having long-standing SOM. ${ }^{11}$ In our preliminary uncontrolled study, performed in children diagnosed with recurrent $\mathrm{AOM}$ and affected by asymptomatic SOM, we have used the well-established probiotic strain $S$. salivarius K12. Strain K12 was administered to children having well-documented middle ear exudate and/or rhinotubaric dysfunction. Our results demonstrate that strain K12: 1) is endowed with a good safety profile when used in otitis-prone children affected by asymptomatic SOM; 2) has a protective effect against $\mathrm{AOM}$ recurrence; and 3) seems to improve some of the clinical outcomes and features relevant in children with SOM. Our study can be criticized for being uncontrolled and for utilizing subjective clinical examination. Nevertheless, 1) this study principally proposed to reevaluate the already well-documented general safety profile of strain $\mathrm{K} 12$, but now, specifically with respect to children with SOM; 2) this was the first time that strain K12 has been administered to evaluate its possible benefit in subjects experiencing middle ear asymptomatic exudate and/or tubaric dysfunction; 3 ) most

Table I Demographic characteristics of the enrolled subjects

\begin{tabular}{|c|c|c|c|c|c|c|c|c|c|}
\hline Sex & Number & Age (yr) & Weight (kg) & Height (m) & BMI $\left(\mathrm{kg} / \mathrm{m}^{2}\right)$ & Allergy & Breastfed & PCVI3 & SBEA \\
\hline Female & 5 & $4.8 \pm 1.0$ & $18.4 \pm 3.3$ & $1.05 \pm 0.07$ & $16.7 \pm 2.9$ & I & 4 & 5 & 0 \\
\hline Male & 17 & $5.8 \pm 2.2$ & $24.2 \pm 6.3$ & $1.14 \pm 0.11$ & $18.5 \pm 2.8$ & 7 & 16 & 15 & 4 \\
\hline Total & 22 & $5.6 \pm 2.1$ & $22.9 \pm 6.2$ & $1.12 \pm 0.11$ & $18.1 \pm 2.8$ & 8 & 20 & 20 & 4 \\
\hline
\end{tabular}

Abbreviations: BMI, body mass index; PCVI3, I3-valent pneumococcal vaccine; SBEA, Streptococcus beta-hemolytic group-A (Streptococcus pyogenes); yr, year. 
Table 2 Clinical outcome in children with secretory otitis media treated by oral route with Streptococcus salivarius KI2

\begin{tabular}{lllll}
\hline & $\mathbf{T}=\mathbf{0}$ & $\mathbf{T = 9 0}$ & $\boldsymbol{\Delta}(\%)^{\mathrm{a}}$ & $\boldsymbol{P}$ \\
\hline AOM incidence $^{\mathrm{b}}$ & 0.40 & 0.23 & 42.5 & $<0.0 \mathrm{I}$ \\
Tone audiometry (right) & $\mathrm{I} .2 \pm 0.6$ & $0.4 \pm 0.5$ & 66 & $<0.0 \mathrm{I}$ \\
Tone audiometry (left) & $\mathrm{I} .1 \pm 0.7$ & $0.4 \pm 0.6$ & 54.6 & $<0.0 \mathrm{I}$ \\
Otoscopy (right) & $\mathrm{I} .5 \pm 0.9$ & $0.9 \pm \mathrm{I} .0$ & 40 & $<0.05$ \\
Otoscopy (left) $_{\text {Endonasal endoscopy }}^{\mathrm{c}}$ & $\mathrm{I} .3 \pm 0.8$ & $0.8 \pm 0.3$ & 38.5 & $<0.05$ \\
Tonsillar examination & $\mathbf{7 0}$ & 50 & 28.6 & $<0.0 \mathrm{I}$ \\
\hline
\end{tabular}

Notes: ${ }^{\mathrm{a}} T=90$ versus $T=0$; ${ }^{\mathrm{b}}$ episodes/month/child; 'reported as average value of the $\%$ of obstruction, statistical analysis has been performed by using arcsin value. Abbreviation: AOM, acute otitis media.

children are not likely to improve spontaneously after having experienced chronic SOM. If the encouraging preliminary results that we have obtained are confirmed in a subsequent double-blind, placebo-controlled study, two hypothesis can be formulated. The first is that the oral treatment with strain K12 may have reduced populations of relevant pathogens in the nasopharyngeal microbiota. Indeed, S. salivarius K12 has been shown to be endowed with strong in vitro inhibitory activity against $S$. pyogenes, $S$. pneumoniae, and $M$. catarrhalis. Moreover, children with recurrent AOM seem to have a relative absence of interfering commensal streptococci in their nasopharyngeal flora. ${ }^{7,8}$ In this study, we did not establish whether alterations in the populations of pathogens occurred. Further trials should include this as an important endpoint. The pathogenesis of SOM is still poorly understood. Analyzed exudates from children with SOM have demonstrated the presence of pathogens in approximately $35 \%$ of cases, by culture techniques, and in approximately $75 \%$ of cases, by polymerase chain reaction. ${ }^{11,27}$ The presence of bacterial pathogens in the exudate could lead to inflammation. Indeed, high levels of proinflammatory cytokines were found in exudates of children with SOM. ${ }^{28}$ S. salivarius K12 has been shown to lower IL-8, a well-known proinflammatory cytokine, in the oral mucosa of colonized subjects. ${ }^{12} \mathrm{~A}$ possible second hypothesis is then that treatment with strain K12 might have stimulated innate immunity, promoting the clearance of bacteria from the middle ear. As a support for this hypothesis, we know that $S$. salivarius

Table 3 Left and right tympanometry in children with secretory otitis media treated by oral route with Streptococcus salivarius KI2

\begin{tabular}{llllllll}
\hline Left & $\mathbf{T = 0}$ & $\mathbf{T = 9 0}$ & $\boldsymbol{P}$ & Right & $\mathbf{T = 0}$ & $\mathbf{T = 9 0}$ & $\boldsymbol{P}$ \\
\hline A & 6 & 14 & $<0.05$ & $\mathrm{~A}$ & 7 & 13 & $<0.05$ \\
B & 9 & 2 & $<0.05$ & $\mathrm{~B}$ & 10 & 2 & $<0.05$ \\
C & 7 & 6 & $\mathrm{~ns}$ & $\mathrm{C}$ & 5 & 7 & $\mathrm{~ns}$ \\
\hline
\end{tabular}

Notes: $\mathrm{A}=$ normal; $\mathrm{B}=$ presence of middle ear exudate; $\mathrm{C}=$ tubaric dysfunction. Abbreviation: ns, not significant.
Table 4 Tolerability, compliance, and side effects in children $(\mathrm{N}=22)$ with secretory otitis media treated for 90 days by oral route with Streptococcus salivarius $\mathrm{KI} 2$ as reported by parents and established by clinician

\begin{tabular}{llll}
\hline & Tolerability & Compliance & Side effects \\
\hline Very good & $\mathrm{N}=13$ & $\mathrm{~N}=16$ & None \\
Good & $\mathrm{N}=7$ & $\mathrm{~N}=4$ & None \\
Acceptable & $\mathrm{N}=2$ & $\mathrm{~N}=2$ & None \\
Unacceptable & $\mathrm{N}=0$ & $\mathrm{~N}=0$ & None \\
\hline
\end{tabular}

Note: $\mathrm{N}=$ number of children.

$\mathrm{K} 12$ is able to reduce colonization by Candida in the oral mucosa and that this effect is not mediated by any type of chemical interference..$^{29}$ This could suggest that stimulation of antibacterial immune effector mechanisms, rather than (or in addition to) bacterial interference, might be responsible for the observed clinical benefits. Both of these hypotheses should now be tested. Originally we also thought that a third possible hypothesis could be drawn to explain the obtained results. It could be that the children with a spontaneous, or caused by the administration of the strain K12, improvement of the adenoid condition also had a clear improvement in terms of exudate presence in the middle ear. We have then decided to statistically analyze the impact of the improvements observed by endonasal endoscopy to possibly correlate them with a parallel and proportional improvement in audiometry, otoscopy, and tympanometry. Statistical results of this analysis (data not shown) demonstrated that no correlation like that could be established. To conclude, our preliminary results have reconfirmed the good safety profile of strain K12 and also seem to indicate that oral treatment with the strain K12 in children with SOM could diminish the amount of fluid in the middle ear and improve some possible hearing problems. This study has to be considered only a starting point to evaluating the role of strain K12 in children with AOM and/or SOM.

\section{Acknowledgments}

The authors wish to thank Dr Risso P for the statistical analysis of the results and Dr Tagg J for the kind suggestions and review of the paper.

\section{Disclosure}

Di Pierro F is the Scientific Director of Velleja Research, the company that developed the finished product tested in this study. The authors report no other conflicts of interest in this work.

\section{References}

1. Tos M. Epidemiology and natural history of secretory otitis. Am J Otol. 1984;5(6):459-462. 
2. Burrows HL, Blackwood RA, Cooke JM, Cooke JM, Harrison RV, Passamani PP; Otitis Media Guideline Team. University of Michigan Health System: Otitis Media Guideline 2013.

3. Loos BG, Bernstein JM, Dryja DM, Murphy TF, Dickinson DP. Determination of the epidemiology and transmission of non-typable Haemophilus influenza in children with otitis media by comparison of total genomic DNA restriction fingerprints. Infect Immun. 1989; 57(9):2751-2757.

4. Rosenfeld RM, Schwartz SR, Pynnonen MA, et al. Clinical practice guideline: tympanostomy tubes in children. Otolaryngol Head Neck Surg. 2013;149(Suppl 1):S1-S35.

5. Gluth MB, McDonald DR, Weaver AL, Bauch CD, Beatty CW, Orvidas LJ. Management of eustachian tube dysfunction with nasal steroid spray: a prospective, randomized, placebo-controlled trial. Arch Otolaryngol Head Neck Surg. 2011;137(5):449-455.

6. American Academy of Family Physicians; American Academy of Otolaryngology-Head and Neck Surgery; American Academy of Pediatrics Subcommittee on Otitis Media with Effusion. Otitis media with effusion. Pediatrics. 2004;113(5):1412-1429.

7. Brook I, Gober AE. In vitro bacterial interference in the nasopharynx of otitis media-prone and non-otitis media-prone children. Arch Otolaryngol Head Neck Surg. 2000;126:1011-1013.

8. Bernstein JM, Faden HF, Dryja DM, Wactawski-Wende J. Micro-ecology of the nasopharyngeal bacterial flora in otitis-prone and non-otitis-prone children. Acta Otolaryngol. 1993;113:88-92.

9. Tano K, Olofsson C, Grahn-Hakansson E, Holm SE. In vitro inhibition of S. pneumoniae, non-typable H. influenzae and M. catarrhalis by alpha-hemolytic streptococci from healthy children. Int $J$ Pediatr Otorhinolaryngol. 1999;47:49-56.

10. Roos K, Hakansson EG, Holm S. Effect of re-colonization with "interfering" alpha streptococci on recurrences of acute and secretory otitis media in children: randomized placebo controlled trial. BMJ. 2001;322: 210-212.

11. Skovbjerg S, Roos K, Holm SE, et al. Spray bacteriotherapy decreases middle ear fluid in children with secretory otitis media. Arch Dis Child. 2009;94(2):92-98.

12. Wescombe PA, Hale JD, Heng NC, Tagg JR. Developing oral probiotics from Streptococcus salivarius. Future Microbiol. 2012;7(12): $1355-1371$.

13. Tagg JR. Prevention of streptococcal pharyngitis by anti-Streptococcus pyogenes bacteriocin-like inhibitory substances (BLIS) produced by Streptococcus salivarius. Indian J Med. 2004;119(Suppl):13-16.

14. Hyink O, Wescombe PA, Upton M, Ragland N, Burton JP, Tagg JR. Salivaricin A2 and the novel lantibiotic salivaricin B are encoded at adjacent loci on a 190-kilobase transmissible megaplasmid in the oral probiotic strain Streptococcus salivarius K12. Appl Environ Microbiol. 2007;73(4):1107-1113.

15. Sharma S, Verma KK. Skin and soft tissue infection. Indian J Pediatr. 2001;68(Suppl 3):S46-S50.
16. Wescombe PA, Burton JP, Cadieux PA, et al. Megaplasmids encode differing combinations of lantibiotics in Streptococcus salivarius. Antonie Van Leeuwenhoek. 2006;90(3):269-280.

17. van Zon A, van der Heijden GJ, van Dongen TM, Burton MJ, Schilder AG. Antibiotics for otitis media with effusion in children. Cochrane Database Syst Rev. 2012;9:CD009163.

18. Power DA, Burton JP, Chilcott CN, Dawes PJ, Tagg JR. Preliminary investigations of the colonisation of upper respiratory tract tissues of infants using a paediatric formulation of the oral probiotic Streptococcus salivarius K12. Eur J Clin Microbiol Infect Dis. 2008; 27(12):1261-1263.

19. Horz HP, Meinelt A, Houben B, Conrads G. Distribution and persistence of probiotic Streptococcus salivarius K12 in the human oral cavity as determined by real-time quantitative polymerase chain reaction. Oral Microbiol Immunol. 2007;22(2):126-130.

20. Burton JP, Wescombe PA, Moore CJ, Chilcott CN, Tagg JR. Safety assessment of the oral cavity probiotic Streptococcus salivarius K12. Appl Environ Microbiol. 2006;72(4):3050-3053.

21. Burton JP, Cowley S, Simon RR, McKinney J, Wescombe PA, Tagg JR. Evaluation of safety and human tolerance of the oral probiotic Streptococcus salivarius K12: a randomized, placebo-controlled, double-blind study. Food Chem Toxicol. 2011;49(9):2356-2364.

22. Di Pierro F, Adami T, Rapacioli G, Giardini N, Streitberger C. Clinical evaluation of the oral probiotic Streptococcus salivarius K12 in the prevention of recurrent pharyngitis and/or tonsillitis caused by Streptococcus pyogenes in adults. Expert Opin Biol Ther. 2013;13(3):339-343.

23. Di Pierro F, Donato G, Fomia F, et al. Preliminary pediatric clinical evaluation of the oral probiotic Streptococcus salivarius K12 in preventing recurrent pharyngitis and/or tonsillitis caused by Streptococcus pyogenes and recurrent acute otitis media. Int J Gen Med. 2012;5: 991-997.

24. Di Pierro F, Colombo M, Zanvit A, Risso P, Rottoli AS. Use of Streptococcus salivarius $\mathrm{K} 12$ in the prevention of streptococcal and viral pharyngotonsillitis in children. Drug Healthc Patient Saf. 2014;6:15-20.

25. Northern JL, Downs MP. Hearing in Children. 3rd ed. Philadelphia, PA: Williams and Wilkins; 1984.

26. Friedman M, Tanyeri H, La Rosa M, et al. Clinical predictors of obstructive sleep apnea. Laryngoscope. 1999;109:1901-1907.

27. Matar GM, Sidani N, Fayad M, Hadi U. Two-step PCR-based assay for identification of bacterial etiology of otitis media with effusion in infected Lebanese children. J Clin Microbiol. 1998;36:1185-1188.

28. Schousboe LP, Ovesen T, Eckhardt L, Rasmussen LM, Pedersen CB. How does endotoxin trigger inflammation in otitis media with effusion? Laryngoscope. 2001;111(2):297-300.

29. Ishijima SA, Hayama K, Burton JP, et al. Effect of Streptococcus salivarius $\mathrm{K} 12$ on the in vitro growth of Candida albicans and its protective effect in an oral candidiasis model. Appl Environ Microbiol. 2012;78(7):2190-2199.
International Journal of General Medicine

\section{Publish your work in this journal}

The International Journal of General Medicine is an international, peer-reviewed open-access journal that focuses on general and internal medicine, pathogenesis, epidemiology, diagnosis, monitoring and treatment protocols. The journal is characterized by the rapid reporting of reviews, original research and clinical studies across all disease areas.

\section{Dovepress}

A key focus is the elucidation of disease processes and management protocols resulting in improved outcomes for the patient. The manuscript management system is completely online and includes a very quick and fair peer-review system. Visit http://www.dovepress.com/ testimonials.php to read real quotes from published authors. 